\title{
LUNAR OCCULTATIONS
}

\author{
From Past to Future Achievements
}

A. RICHICHI

Max-Planck-Institut für Astronomie, Königstuhl 17, D-W-6900 Heidelberg, Germany

\begin{abstract}
For several decades, lunar occultations have represented a powerful and productive technique for high angular resolution investigations of a broad class of astronomical sources: from stellar angular diameters to close binaries, from circumstellar shells to the Galactic Centre.

The resolution and sensitivity offered by lunar occultations still compare favorably with the capabilities of other more modern methods in the optical and near-infrared. A remarkable fact, when one considers that during the past 20 years practically nothing or very little has changed in the way we observe lunar occultations: a sharp contrast with the impressive technological and scientific advancements of other techniques.

The novel possibility of using small areas of fast-readout, low-noise array detectors to record lunar occultations, however, is about to bring radical improvements. We discuss the impact of such detectors on the efficiency of observation and the gain for what concerns the resolution and sensitivity of lunar occultations measurements. New applications such as wavelength-resolved observations and investigations of low-contrast extended sources appear particularly interesting.
\end{abstract}

\section{Introduction}

In a broad sense, lunar occultations are one of the most ancient astronomical techniques: we have written accounts of observations of planets and bright stars being occulted by our satellite dating back to well over 2000 years ago, and even the prehistoric man -although uncapable of keeping a decent logbook by today's standardsmust have wondered as such phenomena. From this point of view, it is certainly remarkable that lunar occultations (LO) are still offering a valuable service to the astronomical community today.

This symposium has illustrated the wealth of techniques which have been developed in recent times to tackle the problem of high angular resolution (HAR) in astronomy. The natural question then is: will LO still serve a purpose, when the forthcoming generation of large ground-based telescopes and telescope arrays will come into operation? Before I begin to assess the present capabilities of LO and try to sketch the future ones, I must apologize for what necessarily will have to be left out of this paper. It is certainly beyond the ability of this author to review the development of LO: in fact, this would be very much a worthy subject for a whole book, and an interesting reading not just for astronomers. Similarly, it is not possible in this short space to cover adequately all the recent results achieved in so many diverse fields of astronomy by means of LO, nor to give a comprehensive description of the data analysis procedures: instead, I will refer to published reviews and representative papers whenever possible. That is not to say that the bibliography here provided is exhaustive: far more complete ones can be found for instance in Böhme (1978), White (1987) and White and Feierman (1987).

The stress here will be mainly on the global apport of LO to the broad area of HAR, and expecially on the near-future improvements. Although LO have found application from the radio to the $\mathrm{X}$-ray range, the present discussion will be restricted mainly to the near-infrared (NIR) applications. 


\section{A Simple Technique for High Angular Resolution}

Observing a lunar occultation amounts to obtaining a fast photometric sequence of a star disappearing (or reappearing) beyond the Moon's limb, with sampling times of $1-10 \mathrm{~ms}$ and durations $\$ 0.5 \mathrm{~s}$. Although not immediately recognized by early supporters of LO (see for instance the interesting exchange between McMahon and Eddington, 1909), the lunar limb acts as a straight edge that diffracts the light from a background star: the high angular resolution (HAR) information is embedded in the fringe pattern, which is rapidly scanned by the telescope as the Moon moves across the sky. Two important facts should be recognized: firstly, the diffraction phenomenon takes place well outside the Earth's atmosphere; and secondly, the telescope aperture plays no role in it. This is the basic reason, why LO can provide HAR results which have outpassed the performance of more modern techniques on the largest telescopes for so many years. The practical success of LO is also due to a lucky combination of the distance and size of the Moon that is seldom fully appreciated. In fact, if our natural satellite had been much closer to us (thus covering more sources in the sky), the occultation phenomena would have been probably too fast even for today's capabilities. In turn, had the Moon been much further away, the events would have been easier to observe, but also very rare. And a bigger Moon would probably have meant dealing with a lunar atmosphere!

It is evident that LO have some major drawbacks: only those sources which happen to lie on the the Moon's orbit can be measured, and even then we have to be ready and running exactly at the time at which the event takes place. The first requisite is not as stringent as it may look: the Moon covers in its orbit about $10 \%$ of the whole sky, and unless you are interested only in a very rare type of objects, chances are that some interesting events will take place in any given period of time. The second requisite is in fact much more of a concern: observations of LO are probably one of the best experiments that one can conceive to prove Murphy's Law: this author has experienced improbable things such as general power failure to a whole district, physical destruction of computer disks, winds in excess of $200 \mathrm{~km} / \mathrm{h}$, and of course a plethora of personal errors that it is not wise to expose in public. If missed, it may be of consolation that the event will be repeated in almost identical circumstances some 18 years later. Finally, a single LO gives just a 1-D scan across a source, and only if the observation can be repeated at other sites or the source is occulted repeatedly (not an uncommon occurrence), can actual 2-D images be reconstructed with procedures similar to that of tomographic imaging.

Given these difficulties, one may be tempted to descard LO as a rather unproductive technique. In fact, there are also several advantages that must be stressed: - telescopes in the 1-2m class suffice for excellent occultation work (although a larger one will clearly yield a better performance). This translates into easy access for the generic observer. This is a major advantage with respect to all other HAR techniques, which require either a large telescope or a unique one of special design. - the instrumentation is particularly simple, requiring a fast photometer as a minimum. Also the data treatment is comparatively fast and direct. A major difference from most other HAR methods is that no calibration source is required.

- in principle, LO are rather unsensitive to atmospheric turbulence and in partic- 
ular to seeing; some lightcurves have been recorded even through clouds.

- at least in the NIR, day-time observations have the same quality of night-time ones (the background issue will be discussed in sect. 4.1).

- finally, LO are the only technique with the rather captivating characteristic of requiring just a few tenths of a second of telescope time!

All these advantages make clear that the disadvantages of LO are satisfactorily counterbalanced. The technique is in fact ideally suited to be scheduled as short interruptions of regular observing time: this ensures that the maximum number of opportunities is used, without much expenditure of telescope time.

\section{Present Scientific Applications and Observational Limits}

Over the years, LO have been used for many different purposes, including investigations of the presence of a lunar atmosphere, studies of lunar motion, and astrometry of radio and X-ray sources. We are concerned here with the HAR applications, in particular in the NIR range. Even with this restriction, it is not possible to offer specific examples in the short available space. Rather, I will try to review the global contribution of LO in some broad areas of research.

\subsection{Angular Diameters and Effective Temperatures}

LO can measure stellar diameters down to 1 mas (see sect. 3.4) with typical accuracies of $0.1-0.2 \mathrm{mas}$. The effective temperature $T_{\mathrm{e}}$ of a star can be determined from its bolometric flux $F$ and the angular diameter $\phi$, according to the definition $F=(\phi / 2)^{2} \sigma T_{\mathrm{e}}^{4}$. Given the fact that stars have angular diameters $\lesssim 0^{\prime \prime} 05$ (and in fact an order of magnitude smaller is more typical even for the brightest cool giants), it can be understood how LO have represented a major source of $T_{e}$ determinations.

The effective temperature is one of the very few quantities characterising a star, that can be measured directly and used as a match for theoretical models. For the coolest spectral types (below $\mathrm{K} 5$ ), the relation between spectrum and $T_{\mathrm{e}}$ was determined for the first time by an analysis based exclusively on LO results (Ridgway et al. 1980). More recently, accurate determinations of angular diameters have been obtained also by interferometry (Di Benedetto and Rabbia 1987, Mozurkewich et al. 1991), with comparable accuracy and resolution. However, LO still represent the only means to reach spectral types beyond M5, which are not well calibrated yet and for which no stars are bright enough to be measured by other techniques.

\subsection{Binary and Multiple Stars}

Multiple stars have represented one of the main applications of LO. A review of this subject can be found for instance in the papers of the IAU Colloquium no. 62 (1981). In this context, the contribution of Prof. Evans, undoubtedly the father of modern LO, is outstanding: an interesting summary is given in Evans et al. (1985). Although it is possible (e.g. Evans et al. 1979) to determine a binary star's orbital parameters only by means of occultations, the nature of LO is more suited to serendipitous discoveries, which can then be followed up where possible by other methods. An illuminating example of this is represented by the surveys of multiplicity at the 
early stages of stellar formation, conducted by means of LO, speckle interferometry and direct imaging in the Taurus and Ophiuchus clouds (Leinert et al. 1992, Simon 1992). The limiting (projected) separation that can be resolved is $\sim 0.005$. At this level of resolution, now attainable also by interferometry, LO still offer unrivalled capabilities of limiting magnitude and dynamic range.

\subsection{Circumstellar Shells and Disks}

Compact circumstellar features such as shells and disks are ideal targets for LO observations, and it is hoped that the numerous results in this area will soon be covered by a dedicated review. The fact that an extended ( $\phi Z 00^{\prime \prime} 04$ in the NIR) source does not produce diffraction fringes has two consequences: the lightcurve is in biunivocal relation to the actual brightness profile, and the natural "clock" provided by the fringes is missing.

It follows that the brightness profiles of extended sources can be recovered in principle as unique solutions, however an uncertainty on the absolute angular scale is intrinsically present (due to the lunar limb slope, and typically $\sim 5 \%$ ). In those cases in which both the central star and the surrounding disk can be observed, this limitation vanishes and rather accurate measurements can be made (see for instance Leinert et al. 1991). For extended sources, the performance of LO is similar to that quoted above for binary stars.

\subsection{Observational Limits of Lunar Occultations}

For what concerns limiting sensitivity, stars as faint as $K \sim 10$ can currently be detected by LO on a large telescope. A more detailed discussion of this aspect, also in connection with future developments, is given in the next section. For what concerns the angular resolution on the other hand, one notes that the smallest angular diameters obtained in practice lie around 1.5mas (see for instance the review by White and Feierman, 1987, which also discusses at length the accuracy of LO). It is natural to ask: is it possible to improve this apparent limit? And: is there an ultimate physical limit to the resolution offered by LO? A full discussion should take into account a realistic and detailed computation of SNR, as well as many other factors. While this would be an interesting study (which apparently has never been undertaken yet), in this limited space we like to give the following simple argument.

Since the number of fringes in a LO lightcurve increases when the diameter decreases, the resolution of LO is ultimately related to the highest fringe number that can be detected above the noise threshold. The fact that LO lightcurves are recorded with practical maximum SNR 100, and then only in favourable cases of intrinsically bright and hence relatively large stars', explains why the above limit is encountered. It would appear that, in order to increase the resolution, we simply need to improve the SNR. This is however practically limited by scintillation. Although methods have been studied (Knoechel and Von Der Heide, 1978), and applied (Richichi et al. 1992), that allow to minimize the lower-frequency part of scintillation, it seems likely that even under optimal conditions the SNR will always be limited to $\ll 10^{3}$, thus preventing major improvements in the resolution. 
A possible application in which simulations show that resolutions in the $0.1 \mathrm{mas}$ range could be obtained, is that of very bright giants of intermediate spectral type, observed through very narrow. filters with $1-2 \mathrm{~m}$ telescopes.

A final consideration prompts us to compare the limiting resolution of LO with that of other HAR techniques. In the latter, the diffraction from the telescope aperture or array baseline is the key factor. In LO the fundamental limit is set by the form of the diffraction fringe pattern, in which the amplitudes scale approximately with $n^{-\alpha}$ ( $n$ being the fringe number). In this universe, $\alpha \sim \frac{1}{2}$ : we can dream of the super-resolution that we could have achieved had $\alpha$ been smaller, but we should also appreciate that it is not much larger!

\section{The Impact of Arrays on Lunar Occultations Performance}

Since the first instrumental observations over 50 years ago (Whitford 1939), LO have experienced relatively little changes. One can identify basically two major steps: the introduction of photoelectric techniques in the optical in the 50's, and of near-IR photometers in the 70's. Occasionally more or less refined variations have appeared such as multi-channel instruments.

The obvious step forward for lunar occultations would be the introduction of panoramic detectors. Attempts in this sense have been made already, but only for specific opportunities and with a limited range of appicability. In particular, one should mention the observations of the Galactic Centre (Simon et al. 1990, Simons et al. 1990), and time- and wavelength-resolved observations of Antares (Schmidtke, in preparation) and Pleione (Gies et al. 1990). The main difficulties have lain with the necessity of read-out rates of $100 \mathrm{~Hz}$ or more, and with the relatively high RON (in the NIR). Even just few years ago, a typical RON was $\sim 1000 \mathrm{e}^{-}$, and values of $30 \mathrm{e}^{-}$were considered only as a nice future possibility (Richichi 1988).

At present, IR arrays have reached a level of performance that makes their use for LO work particularly interesting (possibly by reading out only subframes). To the knowledge of this author, at least two IR cameras are being built at the time of writing, which will support $\mathrm{LO}$ as a standard observing mode.

\subsection{SNR and Limiting Magnitudes}

In order to quantify the limiting magnitude of LO it is necessary, as with any other HAR technique, to specify also a minimum SNR. We define this as the ratio between the signal of the unocculted star, and the standard deviation of the best fit to the data (since the data noise level can be different when the star is in or out of the field). For practical purposes, we shall consider three characteristic values: 1) SNR >50: this is sufficient to exploit fully the capabilities of the technique to measure small angular diameters. 2) SNR 10: inadequate to measure fringes with accuracy, but sufficient to detect binaries with a contrast $\Delta m \lesssim 1.5$, and separations $Z 0$ ".005. 3) finally, we can consider that SNR 1 is roughly the minimum to detect a source at all. A practical demonstration of the data quality as a function of SNR is given by the lightcurves shown in Fig. 1 .

Another necessary constraint is that of integration time $\tau$, which determines of 


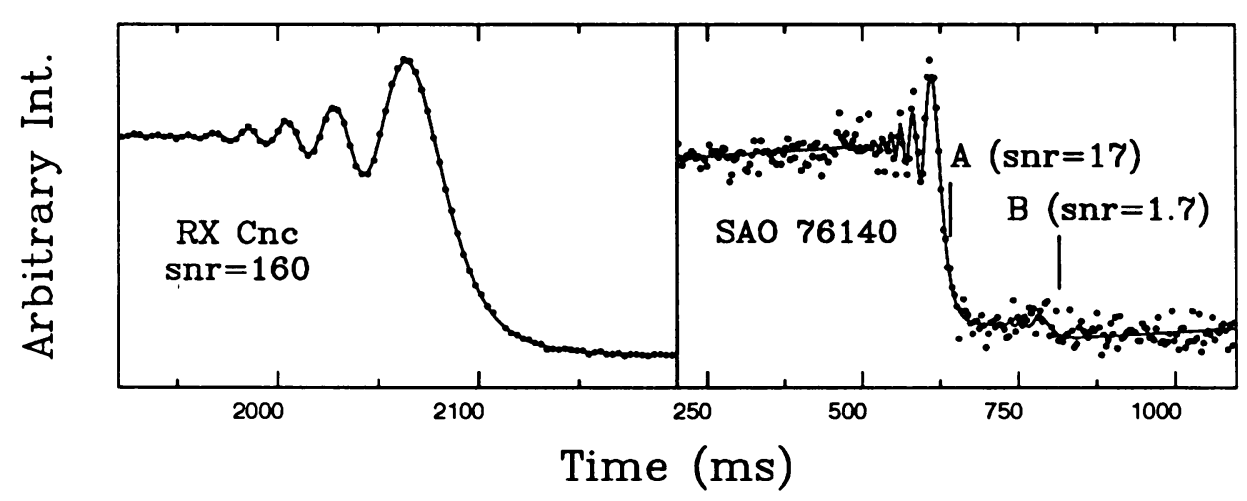

Fig. 1. Quality of LO lightcurves as a function of SNR. At SNR $>100$, the angular diameter of RX Cnc is measured to be $(4.23 \pm 0.09)$ mas. At SNR 1, the $K=7$ companion of SAO 76140 is barely detected. Both lightcurves obtained with the TIRGO $1.5 \mathrm{~m}$ telescope at $2 \mu \mathrm{m}$; data shown as dots, best fits as solid lines.

course the sampling time of the lightcurve. As a rule of thumb, one may consider that $1 \mathrm{~ms}$ in time corresponds to $\sim 0.5$ mas in the sky (although scan rates can get much slower when the events have a high contact angle). We shall consider two characteristic values: 1) $\tau=2 \mathrm{~ms}$ : this is adequate for the best sampling of diffraction fringes. 2) $\tau=12 \mathrm{~ms}$ : this is generally too slow for accurate fringe measurements, but is still sufficient to obtain angular resolutions of 0.005 or better on binary stars and extended sources.

In addition to the obvious importance of telescope size, the sensitivity of LO is strongly dependent on the intensity of the background flux. This latter is extremely variable during an occultation event, being subject at least to the following major factors: phase of the Moon, zenithal distance, position angle of the event and of the terminator, intensity of lunar halo (i.e., water ice crystals in the atmosphere), stray light within telescope and instrument. Some interesting studies have been made, to predict the amount of background due to the scattered moonlight (discussion and references in Stecklum, 1992); however they do not apply in detail to our case, for which it is certainly sufficient to use empirical numbers derived from experience. We shall use the convenient unit of $K$ magnitudes in a $15^{\prime \prime}$ circular diaphragm, and denote this by $\Sigma$. In his experience, this author has observed values ranging from $\Sigma \sim 0$ (day-time observations) to $\Sigma \sim 7$ (close to the intrinsic sky brightness at $2 \mu \mathrm{m}$ ); under more typical conditions, one can assume that $2 \leq \Sigma \leq 5$.

Let us now compare the performance of a) a photometer with a diaphragm $\Omega_{\mathrm{p}}$ and noise $\sigma$, and $b$ ) an array with pixel-size $\Omega_{\mathrm{a}}$ and $\mathrm{RON}=30 \mathrm{e}^{-}$and a well capacity $\sim 2 \times 10^{5} \mathrm{e}^{-}$. Initially, we shall assume that all the signal from the star is detected by one single perfect pixel. Later we shall discuss a more practical situation. The number of photons detected from a source with magnitude $K$ can be expressed as

$$
\mathrm{N}_{(K)} \simeq 10^{(6.84-0.4 K)}\left(\frac{\epsilon}{25 \%}\right)\left(\frac{\Delta \lambda}{0.4 \mu \mathrm{m}}\right)\left(\frac{\tau}{1 \mathrm{~ms}}\right)\left(\frac{D}{4 \mathrm{~m}}\right)^{2}
$$




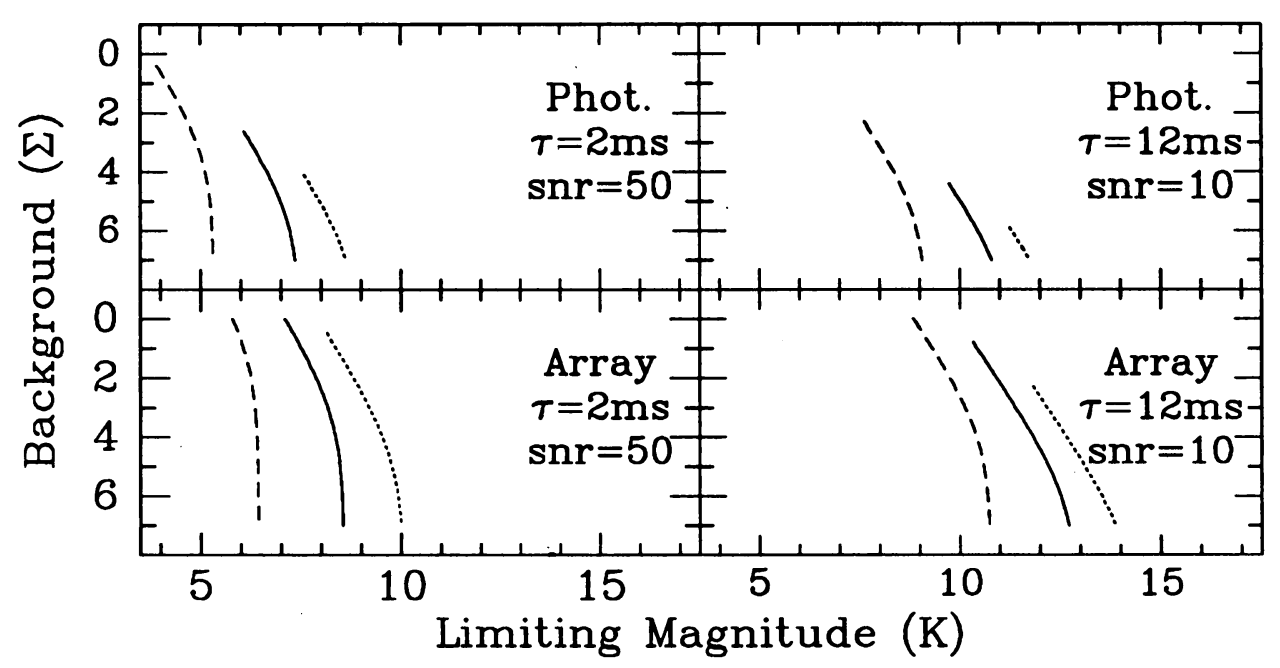

Fig. 2. Limiting magnitudes of LO for an aperture photometer and an array in the NIR. The lines refer to telescopes of $1.5 \mathrm{~m}$ (dashed), $4 \mathrm{~m}$ (solid) and $8 \mathrm{~m}$ (dotted) in diameter.

with the obvious factors. The overall transmission and detection efficiency of telescope and instrument is here denoted by $\epsilon$. Similarly, the number of photons detected from one square-arcsecond of background is

$$
\mathrm{B}_{(\Sigma)} \simeq 10^{(4.59-0.4 \Sigma)}\left(\frac{\epsilon}{25 \%}\right)\left(\frac{\Delta \lambda}{0.4 \mu \mathrm{m}}\right)\left(\frac{\tau}{1 \mathrm{~ms}}\right)\left(\frac{D}{4 \mathrm{~m}}\right)^{2}
$$

The SNR for the two cases above is then computed as follows:

$$
\begin{aligned}
& \text { Photometer } \\
& \left\{\begin{array} { l } 
{ I = ( \mathrm { N } _ { ( K ) } + \Omega _ { \mathrm { p } } ^ { 2 } \cdot \mathrm { B } _ { ( \Sigma ) } ) \cdot \tau } \\
{ N = \sqrt { \sigma ^ { 2 } + I } } \\
{ \mathrm { SNR } \propto \mathrm { A } ^ { \frac { 1 } { 2 } } \cdot \tau ^ { \frac { 1 } { 2 } } }
\end{array} \quad \left\{\begin{array}{l}
I=\left(\mathrm{N}_{(K)}+\Omega_{\mathrm{a}}^{2} \cdot \mathrm{B}_{(\Sigma)}\right) \cdot \tau \\
N=\sqrt{\text { ron }^{2}+\text { dark }+I} \\
\mathrm{SNR} \propto \mathrm{A} \cdot \tau \quad \text { (faint sources) } \\
\mathrm{SNR} \propto \mathrm{A}^{\frac{1}{2}} \cdot \tau^{\frac{1}{2}} \quad \text { (bright sources) }
\end{array}\right.\right.
\end{aligned}
$$

We can neglect the dark current (a safe assumption for the typical integration times with which we are concerned). Fig. 2 illustrates the limiting magnitudes computed, according to eq. 3 , in the two cases of an aperture photometer and of an array. Realistic assumptions based on empirical results have been made for the photometer $\left(\sigma=150 \mathrm{e}^{-}\right.$, saturation at $\sim 4 \times 10^{5} \mathrm{e}^{-}$, diaphragm $\left.\oslash=10^{\prime \prime}\right)$. For the array, a pixel-size of $1^{\prime \prime}$ has been assumed. At least two comments are necessary.

1) In the case of a conventional photometer, one is forced to use a diaphragm which is generally rather large $\left(\oslash>6-10^{\prime \prime}\right)$ because of seeing and telescope pointing. 


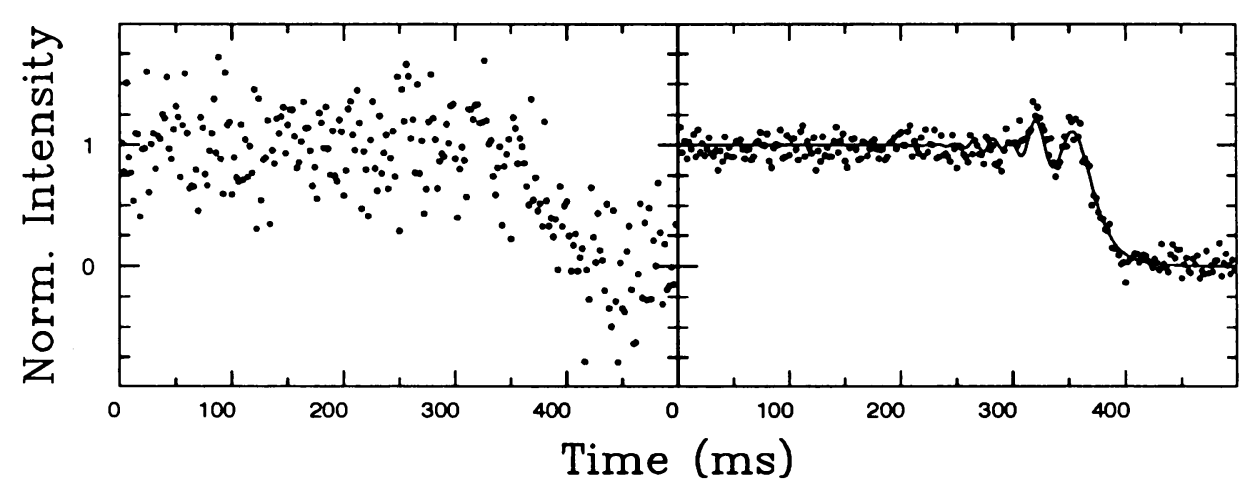

Fig. 3. Simulation of a close binary observed with a photomer (left) and with an array (right). The star has $d=0$.'010, $K=9.0+10.0$ (actual model shown as a solid line).

Therefore, background saturation is a very real limit expecially on large telescopes: this is shown by the truncation in the lines in the upper panels of Fig. 2. It can be seen that integration times $\sim 10 \mathrm{~ms}$ can seldom be used on a $4 \mathrm{~m}$ telescope, and would be practically impossible on an $8 \mathrm{~m}$ one. On the contrary, an array does not only effectively decrease the ratio of background photons to star photons, but it also permits an efficient use under any background condition (including bright limb events in some cases).

2) A (good) photometer is strongly background-limited: this means that increasing telescope area or integration time improves the SNR only by the square root (see eq. 3). Reversely, an array is generally on the limit of BLIP for LO, and can actually be in a RON-limited regime: this is shown by the straight vertical parts of the lines in Fig. 2. Under these conditions, the gain with the increase of area or integration time is linear. It can be appreciated how the magnitude improvements for larger telescopes or longer time is more pronounced in the lower panels of Fig. 2 which refer to the array performance.

It should be noted that, extrapolating the above computations, a detection limit of $K>15$ is obtained for a $4 \mathrm{~m}$ telescope at $\mathrm{SNR}=1$, and this is practically independent of background. We stress that -contrary to the common experiencein LO a SNR 1 is indeed statistically significant, as shown in one example of Fig. 1.

\subsection{Practical Considerations}

The performance of arrays for lunar occultation work has been discussed so far in a slightly abstract approach. In practice, there are several practical considerations that must be kept in mind. In particular, one has to decide the optimal pixel-size $\Omega_{\mathrm{a}}$ as a function of seeing. We cannot enter here the details, however we can mention at least the following points that have to be carefully considered: signal spread over neighbouring pixels; scintillation; photometric accuracy and stability; interpixel gaps. Accurate numerical simulations have been performed, that lead to the following conclusions: a) the optimal choice is $\Omega_{\mathrm{a}} \simeq$ seeing-disk b) the optimal sub- 
array should extend for several seeing-disks across, in order to allow an accurate background and noise estimation in each frame c) the choice of numerical algorithms for the selection of illuminated pixels and fitting of the stellar intensity in each frame is critical: as a minimum, allowance must be made for image wander.

If these conditions are met, simulations have shown that practical performance will match very closely the ideal figures given in the preceding section. As an example, Fig. 3 shows a close binary observed under realistic conditions (seeing $=1.5^{\prime \prime}$, scintillation included) with an aperture photometer and an array on a $3.5 \mathrm{~m}$ telescope. The increase in the data quality is evident.

\section{Scientific Programs for the Future}

The performance offered by the use of NIR arrays has a twofold benefit: for what concerns the traditional fields of investigations by $\mathrm{LO}$, a better sensitivity will be available; at the same time, new applications will become feasible. The following is a list -certainly incomplete- of scientific programs for LO in the near future. The values quoted are for a $4 \mathrm{~m}$ telescope.

- Stellar angular diameters. Paradoxically, this traditional field will be the one less affected by the use of arrays. In fact, the limiting resolution discussed in sect. 3.4 implies that even the coolest stars must have $K<4$ in order to be resolved. This sensitivity is already at the reach of traditional instruments on small telescopes.

- Binary stars. The limit for detection of binaries with $\Delta \mathrm{m} \sim 2$ will be $K \sim 12$, and $K>13$ for those with $\Delta m<0.5$. Particularly attractive in this case is the capability of NIR arrays to operate often also on the bright limb, at least with the shortest integration times. This would effectively yield the true position angle and separation of binaries in just one event, and also the colors if two different wavelengths are used for disappearance and reappearance.

- Brown Dwarfs. The improved sensitivity will open a totally new area for LO, namely the search for low-mass companions of stars in the solar neighbourhood. In a recent catalogue of stars within 25pc from the Sun (Gliese and Jahreiss, 1990), $\sim 400$ are subject to occultations by the Moon: almost all of these have the magnitude and color to be easily observed by LO with an array on a large telescope. For a combined magnitude of $K=10$, LO should allow a detection threshold of $K=13.5$ with resolutions of $0^{\prime \prime} 005$.

- Asteroids. Apart from a few limited measurements by speckle interferometry, HAR investigations of asteroids have traditionally been performed by observing the lightcurve of occulted background stars. While very productive, these events are relatively rare and of difficult prediction, given the small sizes of the occulting objects. Stecklum (1992) has recently considered the occurrence of occultations of asteroids by the Moon. He concludes that, although the method would be very interesting, the number of asteroids bright enough to be observed in the background of a LO in the optical is very small. Also in this field, NIR arrays could open a new area for LO observations. Considering that typical sizes are $\$ 00^{\prime \prime} 05$, and adopting the $V-K$ color of the Sun, one could observe asteroids down to $V \sim 15$; given the high number of objects and the fact that their orbits are almost co-planar with that of the Moon, it follows that these events are of frequent occurrence. 
- AGNs This is one of the most interesting future applications of LO. So far observations of AGNs have been hampered by their relatively low contrast with respect to the background. In the catalogue of Véron and Véron (1991), about 30 quasars and 50 AGNs are subject to occultations, with $B \leq 16$. These objects are very compact, so that a significant fraction of their luminosity is emitted within one seeing-disk, and have tipically red colors: array observations should easily allow resolutions $\sim 10$ times better than that currently provided by HST.

- Wavelength-resolved observations of LO, obtained by dispersing the light so that different parts of the array record different wavelengths, are particularly attractive. The obvious astrophysical advantage is in the fact that the physical size of a source may be strongly dependent on the wavelength even by small shift (i.e. continuum-line emission of stellar atmospheres, AGNs). A second an equally important advantage is that, since diffraction is a chromatic process, lightcurves at two different wavelengths can be stretched to a common scale, and averaged to decrease the (scintillation) noise. This is is an effective means to increase the SNR and thus the resolution of $\mathrm{LO}$ observations.

\section{Acknowledgements}

The author wishes to thank the organizers of this Symposium and the International Astronomical Union for partial support of his participation.

\section{References}

Böhme, D.: 1978, Astron. Nachr., 299, 243.

Di Benedetto, G.P., Rabbia, Y.: 1987, A\&A, 188, 114. 1990, AJ, 100, 1601.

Eddington, A.S.: 1909, . Not. R. Astr. Soc., 69, 178.

Evans, D.S., Fekel, F.C.: 1979, ApJ, 228, 497.

Evans, D.S., Edwards, D.A., Frueh, M., McWilliam, A., Sandmann, W.H.: 1985, AJ, $90,2360$.

Gies, D.R., McKibben, W.P., Kelton, P.W., Opal, C.B., Sawyer, S.: 1990, AJ, 100, 1601.

Gliese, W., Jahreiss, H.: 1990, Preliminary Version of the $3^{\text {rd }}$ Catalogue of Nearby Stars.

Knoechel, G., von der Heide, K.: 1978, A\&A, 67, 209.

Leinert, Ch., Haas, M., Richichi, A., Mundt, R., Zinnecker, H.: 1991, A\&A, $250,407$.

Leinert, Ch., Weitzel, N., Haas, M., Lenzen, R., Zinnecker, H., Christou, J., Ridgway, S.T., Jameson, R., Richichi, A.: 1992, Complementary Approaches to Double and Multiple Star Research, H.A. McAlister and W.I. Hartkopf, eds., p. 21.

McMahon, P.A.: 1909, . Not. R. Astr. Soc., 69, 178.

Mozurkewich., D., Johnston, K.J., Simon, R.S., Bowers, P.F., Gaume, R., Hutter, D.J., Colavita, M.M., Shao, M., Pan, X.P.: 1991, AJ, 101, 2207.

Richichi, A.: 1988, Mem. Soc. Astron. Ital., 58, 431.

Richichi, A., Lisi, F., Di Giacomo, A.: 1992, A\&A, 254, 149.

Ridgway, S.T., Joyce, R.R., White, N.M., Wing, R.F.: 1980, ApJ, 235, 126.

Simon, M., Chen, W.P., Forrest, W.J., Garnett, J.D., Longmore, A.J., Gauer, T., Dixon, R.I.: 1990, ApJ, 360, 95.

Simon, M.: 1992, Complementary Approaches to Double and Multiple Star Research, H.A. McAlister and W.I. Hartkopf, eds., p. 41.

Simons, D.A., Hodapp, K.W., Becklin, E.E.: 1990, ApJ, 360, 106.

Stecklum, B.: 1992, AJ, 104, 1623.

Véron-Cetty, M.P., Véron, P.: 1991, European Southern Observatory Scientific Report, no. 10.

White, N.M.: 1987, Vistas in Astronomy, 30, 13.

White, N.M., Feierman, B.H.: 1987, AJ, 94, 751

Whitford, A.E.: 1939, ApJ, 89, 472. AJ, 94, 751 


\section{Discussion:}

Douglas:

If large enough arrays were available would it not be possible to allow the image to trail across the array during the occultation rather than to have a fast array read out?

\section{Richichi:}

This mode of observation is indeed possible, and two relevant references are given above. The method is very interesting expecially in connection with wavelength dispersion along the unshifted direction. Its general applicability, however, is limited. Consider that, on the average one wants to collect at least $10 \mathrm{~s}$ of data to be sure of catching the event. Even on a $512^{2}$ array, this means sampling times of $\sim 20 \mathrm{~ms}$ at least.

\section{Gurvits:}

Why are only lunar occultations considered as applications of this technique, but not planetary occultations?

\section{Richichi:}

Occultations of stars by planets are certainly very useful - although comparatively rare - events. I did not mention them in my talk, because these observations are best suited to investigating the occulting planet (atmosphere, rings), rather than the occulted star.

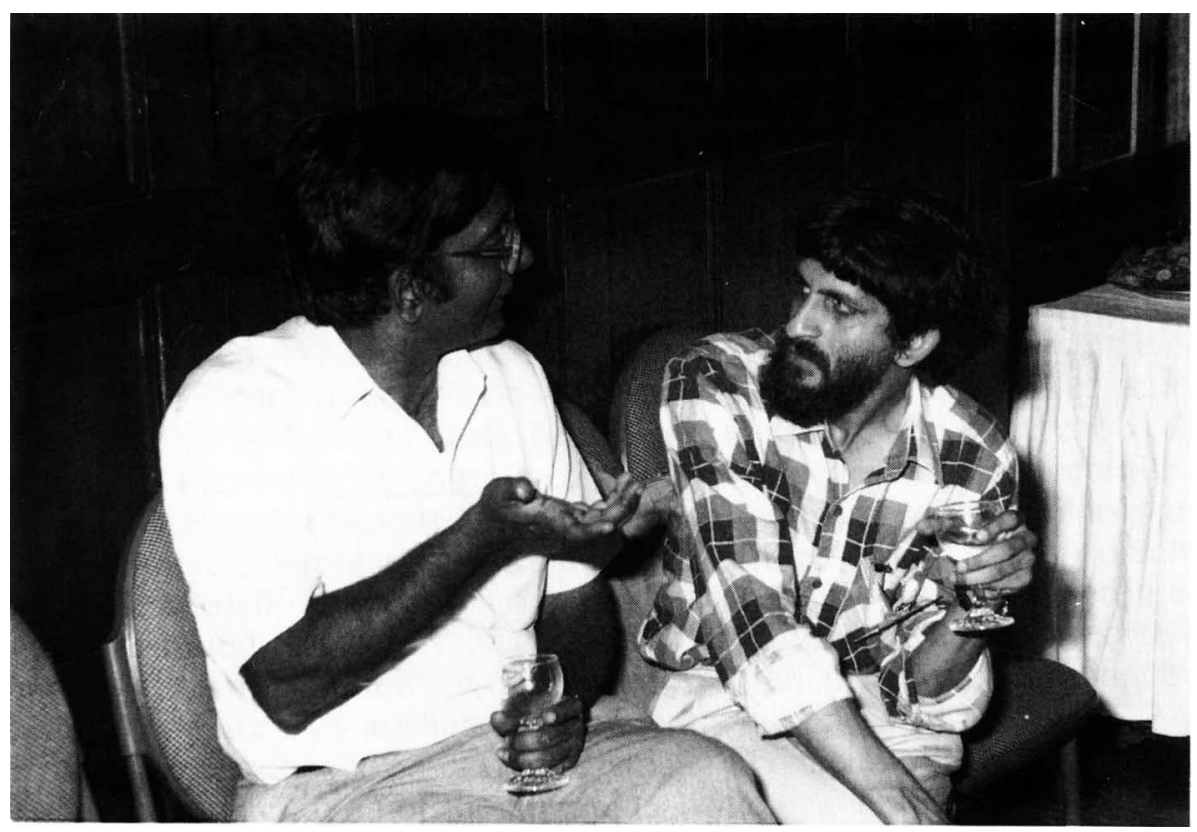

